Bond University

Research Repository

\title{
Attitudes towards anorexia nervosa: Volitional stigma differences in a sample of pre-clinical medicine and psychology students
}

\author{
Bannatyne, Amy Jean; Stapleton, Peta Berenice \\ Published in: \\ Journal of Mental Health
}

DOI:

10.3109/09638237.2016.1149801

Licence:
Other

Link to output in Bond University research repository.

Recommended citation (APA):

Bannatyne, A. J., \& Stapleton, P. B. (2017). Attitudes towards anorexia nervosa: Volitional stigma differences in a sample of pre-clinical medicine and psychology students. Journal of Mental Health, 26(5), 442-448. https://doi.org/10.3109/09638237.2016.1149801

\section{General rights}

Copyright and moral rights for the publications made accessible in the public portal are retained by the authors and/or other copyright owners and it is a condition of accessing publications that users recognise and abide by the legal requirements associated with these rights.

For more information, or if you believe that this document breaches copyright, please contact the Bond University research repository coordinator. 
Running head: ATTITUDES TOWARD ANOREXIA NERVOSA

Attitudes Toward Anorexia Nervosa: Volitional Stigma Differences in a Sample of PreClinical Medicine and Psychology Students

Amy Jean Bannatyne*1 and Peta Berenice Stapleton ${ }^{1}$

${ }^{1}$ Bond University, Department of Psychology (14 University Dr, Robina, QLD Australia, 4229)

* Corresponding Author

Email: abannaty@bond.edu.au

Contact telephone number: +61755952515

Facsimile: +61755952540

\section{Declaration of Interests}

There are no known conflicts of interest associated with this publication and there has been no financial support for this work that could have influenced its outcome. 
Running head: ATTITUDES TOWARD ANOREXIA NERVOSA

This is an Accepted Manuscript of an article published by Taylor \& Francis Group in Journal of Mental Health on 29 Feb 2016, available online: https://doi.org/10.3109/09638237.2016.1149801. 


\begin{abstract}
Background: Anorexia nervosa (AN) is a highly stigmatised condition, with treatment often involving multidisciplinary care. As such, understanding and comparing the attitudes of emerging mental health and medical professionals toward AN, within the content of sexbased differences, is pertinent to facilitate the development of targeted stigma interventions.

Aims: Examine the volitional stigmatisation of AN in emerging medical and mental health professionals.
\end{abstract}

Method: Participants $(N=126)$ were medical $(n=41)$ and psychology students $(n=85)$ who completed a range of attitudinal outcome measures (e.g., Causal Attributions Scale, Eating Disorder Stigma Scale, Opinions Scale, Characteristics Scale, and Affective Reaction Scale). Results: Across both disciplines, men were found to exhibit significantly higher ED stigma, considered AN to be a more trivial and weak illness, and attributed greater levels of blame and responsibility to AN sufferers. Men also had significantly lower biogenetic causal attributions. Compared to psychology students, medicine students exhibited slightly greater anticipation of negative reactions in response to $\mathrm{AN}$, obtained higher selfish/vain scores, and considered sociocultural factors to contribute "a lot" in the development and maintenance of AN.

Conclusions: Overall, results indicate interventions aimed at improving ED mental health literacy are needed, specifically targeting males and potentially medical students.

Keywords: eating disorders, anorexia nervosa, volitional stigma, health professionals, psychology, medicine 
Attitudes Toward Anorexia Nervosa: Volitional Stigma Differences in a Sample of PreClinical Medicine and Psychology Students

Over the past decade, the concept of mental illness stigma has attracted significant attention from researchers, health professionals, policy makers, and the general population. Stigma is a social process characterised by exclusion, blame, rejection, or devaluation (Goffman, 1963), which can result from the experience or anticipation of adverse social judgment. This judgment is typically deeply discrediting and distressing (Weiss \& Ramakrishna, 2006). For individuals suffering from an ED, particularly anorexia nervosa (AN), stigma and discrimination are an unfortunate and pervasive reality, inflicted by both the general population and health profession (see Thompson-Brenner, Satir, Franko, \& Herzog, 2012, for a full review).

Stigma in relation to AN typically manifests in the perception of AN as a voluntary or self-inflicted illness, often referred to as "volitional stigma". However, unlike traditional mental illness stigma, which proposes individuals are set apart from "normals", volitional stigma reportedly involves judgment by normal behavioural standards (Easter, 2012), possibly due to the ego-syntonic nature of AN. That is, individuals (i.e., friends, family members, treating professionals) may recognise the severity of AN, yet consider some forms of disordered eating behaviour (e.g., strict dietary and weight control) to be desirable or admirable (Geerling \& Saunders, 2015; Griffiths, Mond, Murray, \& Touyz, 2015; Mond, Robertson-Smith, \& Vetere, 2006). The consequences of this stigma are well documented, particularly the low rate of treatment seeking among this patient population for ED-related concerns (Griffiths, Mond, Murray, \& Touyz, 2014; Griffiths et al., 2015a; Touyz, 2011) and other non-ED medical ailments (Bannatyne \& Stapleton, in press; de la Rie, Noordenblos, Donker, \& van Furth, 2006).

The attitudes and reactions of health professionals are an important component of stigma; yet, health professionals have only recently become the focus of stigma research. 
Previously, health professionals were expected to have more favourable attitudes due to their level of knowledge and mental health literacy, in addition to more frequent contact with those suffering from mental illness (Lauber, Anthony, Ajdacic-Gross, \& Rössler, 2004). However, research over the past decade has indicated there is little, if any, distinction between public and professional beliefs regarding mental illness (Bannatyne \& Stapleton, 2015; Jorm, 2000).

In relation to $\mathrm{AN}$, clinicians across a spectrum of health disciplines, particularly at entry-level, have been shown to frequently report negative reactions in response to sufferers (Franko \& Rolfe, 1996). For example, first-year residents in medicine, psychiatry, and paediatrics, have been found to experience greater negative affect (e.g., anger, irritation, and hostility) when treating patients with AN compared to patients with other physical and psychological conditions (Brotman, Stern, \& Herzog, 1984; Fleming \& Szmukler, 1992). Emotional responses have also been revealed in samples of psychologists and psychiatrists (e.g., Jones, Saeidi, \& Morgan, 2013), with one study (e.g., Burket \& Schramm, 1995) demonstrating 31 percent of participants $(N=90)$ reported a preference not to treat ED patients due to frustration (87\%) and anger (63\%).

Extensive research has indicated negative attitudes toward particular mental illnesses develop early in an individual's education and training (Williams \& Leichner, 2006). In relation to AN, medical students and early-career physicians, who have little to no experience in treating EDs, often report a lack of empathy and strongly endorse beliefs of self-infliction (Walker \& Lloyd, 2011). This is of interest given the strong endorsement of the biomedical approach within the medical profession and the wealth of literature highlighting the contribution of multiple factors (e.g., biogenetic, psychological, and social) in the development and maintenance of AN (see Culbert, Racine, \& Klump , 2015, for a full review). However, given the multifactorial and interdisciplinary nature of treating AN (e.g., physical and psychological intervention), understanding the attitudinal views of emerging mental health professionals (e.g., psychologists), and comparing these to emerging medical 
professionals, is pertinent to facilitate the development of targeted stigma alleviation efforts. Previous research has revealed psychology students do endorse blame and vanity-related stigma toward AN, especially when presented with a sociocultural explanation of the condition (Bannatyne \& Abel, 2014; Crisafulli, Thompson-Brenner, Franko, Eddy, \& Herzog, 2010).

Evidence concerning attitudinal sex differences toward EDs in health professionals is, however, surprisingly absent. This is of interest, as although women are highly prevalent within healthcare, particularly in nursing and allied health roles, there appears to be a greater ratio of male physicians (Gupta, Diallo, Zurn, \& Dal Poz, 2003). Understanding the attitudes of these physicians is potentially important in removing treatment barriers, thereby enhancing treatment seeking in EDs. Existing research with university students, including psychology students, has indicated young men consider AN to be a condition of lesser severity than young women (Mond \& Arrighi, 2011; Wingfield, Kelly, Serdar, Shivy, \& Mazzeo, 2011), perceive ED characters as less likeable (Wingfield et al., 2011), are more likely to stigmatise EDs (Griffiths et al., 2014), and are less likely to approach sufferers (Griffiths et al., 2014). However, other studies have suggested men and women are equivalent in their knowledge and attitudes toward EDs (Hunt \& Rothman, 2007).

Accordingly, the empirical objective of the current study was to examine and compare the attitudes and beliefs of psychology and medicine students toward AN, in addition to exploring sex-based differences within and between disciplines. Based on previous research (e.g., Bannatyne \& Stapleton, 2015) and the general presumption psychology students have received greater educational exposure to mental illness, it was hypothesised that psychology students would be significantly less likely to blame and stigmatise AN compared to medicine students. That is, psychology students would exhibit lower levels of ED stigma, fewer negative attitudes, more positive affect, and lower self-infliction beliefs. Given the nature of medical education, it was anticipated medical students would exhibit greater endorsement of 
biogenetic etiological factors compared to psychology students. No significant difference was expected between groups on sociocultural causal attributions; however, sociocultural factors were expected to be the most salient explanation for AN across all participants. No specific hypotheses in relation to the sex-based disciplines differences were developed.

\section{Method}

\section{Participants}

One hundred and twenty six Medicine and Psychology students were recruited through the Medicine and Psychology departments at the authors' institution. Medicine students had just commenced their fourth year of study, which involved active contact in health settings. Psychology students were in their final year of an undergraduate psychological science degree or involved in a fourth-year post-graduate degree (i.e., honours or post-graduate diploma). Consistent with the reasoning of Crisafulli, Von Holle, and Bulik (2008), that previous experiences of the illness may impact etiological perceptions and attitudes, 12 female participants $(9.5 \%)$ with a self-reported history of AN were removed from the dataset. The final sample comprised of 114 medical and psychology students aged 19 to 55 years $(M=23.39, S D=5.10)$, with no self-reported history of AN. Participant demographics can be seen in Table 1. All participants gave informed consent. The research was approved by the ethics committee at the authors' institution.

\section{INSERT TABLE 1 HERE}

\section{Materials}

Demographic Questions. Participants were asked to supply demographic information such as age, sex, ethnicity and year of degree, for the purpose of describing the sample. Each participant was also asked to indicate whether or not he/she felt, or had been told by a health professional, he/she had suffered from AN. This wording was intended to account for participants who had recovered from AN, as well as participants that may have struggled with AN, but never received a formal diagnosis. 
Causal Attributions. Participants were asked to indicate on a 7-point Likert scale $(1=$ does not contribute at all to $7=$ main contributing factor) the extent to which nine different factors contribute to the development of AN. In line with previous research (e.g., Bannatyne \& Abel, 2015), the Causal Attributions Scale (Crisafulli et al., 2008) was separated into two subscales for analysis: biogenetic attributions and sociocultural attributions. An average score for each subscale was created for analysis. Previous research (e.g., Bannatyne \& Abel, 2015) has reported good internal consistencies ranging from .82 to .86 for the sociocultural subscale and .83 to .84 for the biogenetic subscale. Reliability analyses for the current study revealed similar internal consistencies (.84 and .85 , respectively).

Opinions. Participants' attitudes toward individuals with AN were assessed using the Opinions Scale (Stewart, Keel, \& Schiavo, 2006). Participants were asked to indicate their degree of agreement with five stigmatising statements on a 5-point Likert scale $(1=$ strongly disagree to $5=$ strongly agree). The five items reflected stigmata such as responsibility (e.g., "are to blame for their condition"), as well as fear and exclusion (e.g., "are a danger to others"). Higher scores were indicative of more negative opinions. The Opinions Scale was analysed at a subscale level, with average scores for each subscale created for analysis. Previous research (e.g., Bannatyne \& Abel, 2015) has reported internal consistencies of .68 for Fear and Exclusion, and .86 for Responsibility. Reliability analyses for the current study revealed slightly lower internal consistencies (.67 and .79 , respectively).

ED Stigma. Participants were asked to complete the Eating Disorder Stigma Scale (Crisafulli et al., 2010), a self-report measure designed to assess a variety of beliefs people may hold about AN. Participants were asked to indicate the extent to which they agreed with each statement on a 5 -point Likert scale $(1=$ strongly disagree to $5=$ strongly agree $)$. The ED Stigma Scale contained 20-items reflecting stigmata such as trivialisation, selfish/vain, weak, and blame. Research has indicated the ED Stigma Scale is a psychometrically sound instrument with internal consistencies ranging from .95 for the full scale, and .80 to .92 for 
the subscales (Bannatyne \& Abel, 2015, Bannatyne \& Stapleton, 2015; Crisafulli et al., 2010). For the purpose of the current study, the ED Stigma Scale was analysed at the full scale and subscale level, with reliability analyses revealing high internal consistencies for the full scale (.94) and subscales (.89 to .94$)$.

Characteristics Assigned to AN. Participants were asked to rate individuals with AN on various personality and behavioural attributes using the Characteristics Scale (Penn et al., 1994), which has been found to have a good internal consistency of .85 to .87 (Bannatyne \& Abel, 2015; Penn et al., 1994). The Characteristics Scale consists of 20 items, each of which is an opposing dichotomous adjective pair (e.g., intelligent/unintelligent). Participants were asked to indicate the extent to which the adjectives reflected characteristics of individuals with AN on a 7-point Likert scale, with one indicating high perceived levels of the positive adjective, four indicating neutral, and seven indicating high perceived levels of the negative adjective. An average score was created for analysis. Reliability analyses revealed an internal consistency of .88 , similar to previous research.

Emotional Response to AN. Participants were asked to indicate how they thought they would feel should they interact with an individual suffering from AN using the Penn et al. (1994) Affective Reaction Scale. This self-report scale consisted of 10 dichotomous adjective pairs (e.g., empathic/disgusted) containing emotive content. Participants rated each item on a 7-point Likert scale with one indicating positive emotionality, four acting as neutral, and seven indicating negative emotionality. An average score was created for analysis. Previous research has indicated the Affective Reaction Scale has good internal consistency ranging from .86 to .87 (Bannatyne \& Abel, 2015; Penn et al., 1994), with reliability analyses in the current study revealing an internal consistency of .86.

\section{Procedure}

Psychology students were recruited via an information sheet on a university research board. Students interested in participating were asked to contact the first author via email for 
the link to the online questionnaire. Medicine students were recruited as part of an educational intervention trial delivered during a clinical paediatrics rotation (See Bannatyne \& Stapleton, 2015). The current study utilised the pre-intervention data from this trial. All participants were asked to read an explanatory statement, provide informed consent, and complete a questionnaire package consisting of demographic questions and items from the dependent variables. Completion of the questionnaire took approximately 20 minutes. Contact information for counselling services at various locations was provided and participants were given the option to have their responses withdrawn.

\section{Statistical Plan}

The data were analysed using SPSS Version 22. An alpha level of .05 was utilised to determine the statistical significance of all results. Due to the clustering of bivariate correlations, three between-subjects multivariate analyses of covariance (MANCOVA) were performed. The first MANCOVA was performed on stigmatisation outcomes (blame, trivialisation, weak, selfish/vain, responsibility, and fear and exclusion). The second MANCOVA was performed on attitudinal outcomes (characteristics and affective reaction). The third MANCOVA was performed on causal attributions (sociocultural and biogenetic). Due to a high level of multicollinearity with the ED Stigma Scale subscales, the total ED stigma scale score was evaluated in a two-way ANCOVA.

\section{Results}

\section{Preliminary Analyses}

Preliminary analyses revealed there were significant differences between the groups on $\operatorname{sex} \chi^{2}(1)=28.89, p=<.001$ and age $t(112)=2.17, p=.032$. As such, age was run as a covariate. The means and standard deviations for all measures can be seen in Table 2 .

\section{INSERT TABLE 2 HERE}

\section{Stigmatisation MANCOVA}


With the use of Wilk's criterion, a significant multivariate main effect of the sex of particiapnts was revealed $F(6,105)=2.09, p=.034$, partial $\eta^{2}=.13$, after controlling for the effect of age. The main effect of degree type failed to reach significance $(p=.061)$, as did the interaction between degree and participants' sex $(p=.316)$. As a result, subsequent analyses focused primarily on the main effect of participants' sex; however, the main effect of degree type was examined for exploratory purposes.

As can be seen in Table 2, univariate analyses revealed a significant effect of sex on blame, weak, trivialisation, and responsibility. That is, across both disciplines men were found to have higher blame, responsibility, weak, and trivialisation scores than women. No significant effects of participants' sex were observed for fear and exclusion, or selfish/vain scores. However, a significant main effect of degree type was observed on the selfish/vain subscale, with medicine students observed to have significantly higher scores on the selfish/vain subscale than psychology students.

\section{Total ED Stigma ANCOVA}

A two-way ANOVA was conducted to examine the effects of degree type and participants' sex on total ED stigma. The interaction effect between degree type and participants' sex was not statistically significant $(p=.361)$, in addition to the main effect of degree type $(p=.173)$; however, a significant univariate effect of participants' sex was revealed $F(1,114)=7.02, p=.009$, partial $\eta^{2}=.06$, after controlling for the effects of age. That is, males across both degrees had significantly higher ED stigma scores. While adjusted mean scores revealed medicine students obtained higher ED stigma scores (mean difference of 4.5 points), this difference was not statistically significant.

\section{Attitudinal MANCOVA}

With the use of Wilk's criterion, a significant multivariate effect of degree type was 
revealed $F(2,109)=3.46, p=.044$, partial $\eta^{2}=.07$, after controlling for the effects of age. A significant univariate effect for affective reaction was observed $F(1,112)=7.19, p=.008$, partial $\eta^{2}=.06$. The multivariate main effect of participants' sex failed to reach significance $(p=.973)$, as did the interaction between degree and participants' sex $(p=.331)$. At the univariate level, degree type was found to have a significant effect on affective reaction $F(1$, $110)=3.86, p=.038$, partial $\eta^{2}=.06$, with medicine students reporting more negative emotional responses toward AN sufferers than psychology students. No univariate effect was revealed for characteristics $(p=.416)$.

\section{Causal Attributions MANCOVA}

After controlling for the effects of age, a significant multivariate effect was revealed for the sex of participants $F(2,109)=4.54, p=.017$, partial $\eta^{2}=.08$. The multivariate main effect of degree type failed to reach significance $(p=.704)$, as did the interaction between degree and participants' sex $(p=.597)$. At the univariate level, significant differences were observed between men and women for sociocultural attributions $F(1,110)=6.52, p=.012$, partial $\eta^{2}=.06$, and biogenetic attributions $F(1,110)=5.26, p=.049$, partial $\eta^{2}=.05$. Across both disciplines, men demonstrated significantly lower endorsement of biogenetic factors, while women demonstrated significantly higher endorsement of sociocultural factors.

\section{Discussion}

This study aimed to investigate and compare medicine and psychology students stigmatising attitudes and beliefs about AN within the context of sex differences. It was hoped results of the current study may reveal specific groups who require additional education and training in relation to $\mathrm{AN}$, in addition providing direction for the development of sex-specific stigma interventions.

\section{Differences between Medicine and Psychology Students}

While medicine students appeared to obtain higher scores on numerous ED stigma domains (e.g., blame, trivialisation, weak, responsibility, selfish/vain, and total ED stigma), 
these differences was only statistically significant for the selfish/vain subscale. Medicine students also obtained higher affective reaction scores, indicative of slightly more negative emotional responses, compared to psychology students who obtained scores indicative of neutral emotional responses. This finding was consistent with the result of previous research which highlight the less than positive attitudes many entry level clinicians enter into the clinical environment holding in response to AN, particularly feelings of frustration and irritation (Brotman et al., 1984). In the current study, 25 and 13 percent of medical students experienced feelings of irritability and anger, respectively. While just under half experienced feelings of anxiety and apprehension (40\% and 35\%, respectively), possibly reflective of the perceived inadequacy of medical school training reported in literature (Walker \& Lloyd, 2011).

However, of greater concern, almost three quarters of medical students (70\%) reported feelings of resent in relation to treating $\mathrm{AN}$, possibly due to the low prestige of $\mathrm{AN}$ compared to other illnesses (e.g., physical conditions). Previous research has demonstrated hospital specialists, primary care physicians, and medical students consistently rank AN as an illness of low prestige (Album \& Westin, 2008), likely due to perceptions of AN as a chronic illness, with a relatively poor treatment prognosis until the sufferer "chooses", or motivates him/herself, to respond to treatment (Bannatyne \& Stapleton, 2015; Currin, Waller, \& Schmidt, 2009). However, psychology students also reported feelings of anxiety (54\%), apprehension (43\%), and tension (39\%), likely due to the lack of clinical contact at third and fourth year levels, or poor ED knowledge; but, feelings of irritation (11\%), anger (5\%), and resent (5\%) were lower than medical students.

Interestingly, a quarter of participants in both samples reported feelings of disgust. Possibly this response is due to the visual manifestation of AN (i.e., emaciation), consistent with the aesthetic and concealability stigma dimensions proposed by Jones et al. (1984). This response of disgust does, however, have the potential to damage therapeutic relationships 
given sufferers are often acutely sensitive to social judgment (Hepworth \& Paxton, 2007). It would be of interest to compare the reaction to AN sufferers of varying BMI's and different EDs (e.g., AN vs BN) to determine the origin of this disgust. Regardless, the findings highlight the need for greater ED education incorporating self-reflection and management of transference at an undergraduate level. Previous research has demonstrated the benefit of brief educational interventions with medical students in relation to AN (Bannatyne \& Stapleton, 2015). Similar interventions may be useful for psychology students, particularly males.

Although no significant differences in sociocultural and biogenetic attribution scores were observed between medicine and psychology students, it is curious that medicine students perceived sociocultural factors (e.g., media pressure, vanity, thin ideals) to contribute "a lot" in the development of AN, particularly given the structure of medical education which emphasises biomedical factors and the wealth of literature available highlighting the contribution of multiple factors in the development of AN. As we note elsewhere (e.g., Bannatyne \& Stapleton, 2015), this raises questions as to the nature of ED education received, transference of attitudes/beliefs in relation to $\mathrm{AN}$, and/or the extent to which students evaluate AN based on personal assumptions developed from popular media representations, which are often inaccurate.

\section{Sex-based differences}

Possibly of greater interest is the impact participants' sex had on the stigmatisation of AN. Across both disciplines, men were found to exhibit significantly higher ED stigma scores, considered AN to be a more trivial and weak illness, attributed greater levels of blame and responsibility, and had significantly lower biogenetic causal attributions. These findings potentially highlight male students in both medicine and psychology are at increased risk of developing stigmatising beliefs about a condition that predominately affects females, which could impact clinical care and therapeutic alliances. While the direction of sex differences 
was not hypothesised, this finding is consistent with previous research suggesting men generally perceive AN to be less severe, self-inflicted, and trivial (Griffiths et al., 2014; Mond \& Arrighi, 2011; Wingfield et al., 2011), while women exhibit greater envy and admiration (Borenstein, 2011; Mond et al., 2006); however, levels of envy were not assessed in the current study.

Other researchers (e.g., Stewart et al., 2006) have also demonstrated female students are less likely to view mental illness negatively compared to male peers. Given AN is an illness which primarily afflicts young women, it is possible a greater level of sympathy was present, which may explain the revealed sex differences. Some researchers have suggested men may stigmatise and underestimate the chronicity and impact of AN as they are less likely to experience or be directly affected by the condition (Wingfield et al., 2011). Conversely, Crisp (2005) proposed perceived vulnerability to developing a specific illness could actually increase discomfort and stigma (e.g., perceptions of affected individuals being "weakwilled"). This suggestion would predict women in the current study to have exhibited higher weak subscale scores; however, this pattern did not emerge, suggesting further research is required to understand sex-based differences in volitional stigma using larger samples.

The lower endorsement of biogenetic factors by male participants could also potentially explain the greater level of volitional stigma observed in males (i.e., biogenetic factors are associated with feelings of sympathy and greater helping behaviours due to the perception these factors are not in the individual's control). This potentially indicates male students, in particular, may benefit from interventions emphasising biogenetic and neurobiological factors that can lead to the onset and maintenance of AN to decrease perceptions of personal responsibility and reduce trivialisation beliefs through medicalised discourse.

\section{Limitations and Future Directions}


Although the current study has revealed interesting findings regarding sex- and discipline-based influences on the volitional stigmatisation of AN, certain limitations are noted. These limitations include the small sample size, which may have reduced statistical power to determine significant differences; limited generalisability due to recruitment from a single institution; and the self-report nature of data, which is vulnerable to social desirability concerns and does not measure actual behavioural responses. The fact that 11 of the 12 excluded female participants who indicated a lifetime history of AN were derived from the psychology student sample also possibly indicates the level of ED symptomatology was higher in the psychology sample, potentially confounding the results (i.e., the lower levels of stigma observed in female psychology students may be the result of empathy due to greater ED psychopathology, rather than specific discipline differences). Future research should seek to measure ED symptoms of samples to control for this.

Overall, results of the current study tentatively suggest male medical and psychology students are prime candidates for ED mental health literacy interventions, particularly those that incorporate biogenetic information within a biopsychosocial framework to reduce perceptions of self-infliction and vanity. However, additional research exploring sex-based differences in stigma traits is needed to assist with the development of sex-specific stigma interventions to facilitate positive interaction with ED sufferers', thereby reducing treatment barriers and improving patient outcomes.

\section{References}

Album, D., \& Westin, S. (2008). Do diseases have a prestige hierarchy? A survey among physicians and medical students. Social Science \& Medicine, 66, 182-188. doi:10.1016/j.socscimed.2007.07.003

Bannatyne, A. J., \& Abel, L. M. (2015). Can we fight stigma with science? The effect of 
etiological framing on attitudes toward anorexia nervosa and the impact on volitional stigma. Australian Journal of Psychology, 67, 38-46. doi:10.1111/ajpy.12062

Bannatyne, A., \& Stapleton, P. (2015). Educating medical students about anorexia nervosa: A potential method for reducing the volitional stigma associated with the disorder. Eating Disorders: The Journal of Treatment and Prevention, 2, 115-133. doi:10.1080/10640266.2014.976102

Bannatyne, A. J., \& Stapleton, P. B. (in press). Eating disorder patient experiences of volitional stigma within the healthcare system and views on biogenetic framing: A qualitative perspective. Australian Psychologist.

Borenstein, B. (2011). The effect of biological versus environmental causal explanations on the stigmatisation of major depression and anorexia nervosa. (Unpublished Psychology Honours). Connecticut College, New London, Connecticut.

Brotman, A., Stern, T., \& Herzog, D. (1984). Emotional reactions of house officers to anorexia nervosa, diabetes, and obesity. International Journal of Eating Disorders, 3, 71-77. doi:10.1002/1098-108X(198422)3:4<71::AID-EAT2260030409>3.0. $\mathrm{CO} ; 2-\mathrm{O}$

Burket, R. C., \& Schramm, L. L. (1995). Therapists' attitudes about treating patients with eating disorders. Southern Medical Journal, 88, 813-818. doi:10.1097/00007611199508000-00004

Crisafulli, M. A., Thompson-Brenner, H., Franko, D. L., Eddy, K. T., \& Herzog, D. B. (2010). Stigmatisation of anorexia nervosa: Characteristics and response to intervention. Journal of Social and Clinical Psychology, 29, 756-770. doi:10.1521/jscp.2010.29.7.756

Crisafulli, M. A., Von Holle, A., \& Bulik, C. M. (2008). Attitudes towards anorexia nervosa: The impact of framing on blame and stigma. International Journal of Eating Disorders, 41, 333-339. doi:10.1002/eat.20507 
Crisp, A. H. (2005). Stigmatization of the discrimination against people with eating disorders including a report of two nationwide surveys. European Eating Disorders Review, 13, 147-152. doi:10.1002/erv.648

Culbert, K. M., Racine, S. E., \& Klump, K. L. (2015). Research review: What we have learned about the causes of eating disorders - a synthesis of sociocultural, psychological, and biological research. Journal of Child Psychology and Psychiatry (early access view). doi:10.1111/jcpp.12441

Currin, L., Waller, G., \& Schmidt, U. (2009). Primary care physicians' knowledge of and attitudes toward the eating disorders: Do they affect clinical actions? International Journal of Eating Disorders, 42, 453-458. doi:10.1002/eat.20636

de la Rie, S., Noordenbos, G., Donker, M., \& van Furth, E. (2006). Evaluating the treatment of eating disorders from the patient's perspective. International Journal of Eating Disorders, 39, 667-676. doi:10.1002/eat.20317

Easter, M. M. (2012). "Not all my fault": Genetic, stigma, and personal responsibility for women with eating disorders. Social Science \& Medicine, 75, 1408-1416. doi:10.1016/j.socscimed.2012.05.042

Fleming, J., \& Szmukler, G. I. (1992). Attitudes of medical professionals towards patients with eating disorders. Australian and New Zealand Journal of Psychiatry, 26, 436443. doi:10.3109/00048679209072067

Franko, D. L., \& Rolfe, S. (1996). Countertransference in the treatment of patients with eating disorders. Psychiatry, 59, 108-116.

Geerling, D. M., \& Saunders, S. M. (2015). College students' perceptions of individuals with anorexia nervosa: Irritation and admiration. Journal of Mental Health, 24, 1-5. doi:10.3109/09638237.2014.998807

Goffman, E. (1963). Stigma: Notes on the management of spoiled identity. Englewood Cliffs, NJ: Prentice Hall. 
Griffiths, S., Mond, J. M., Li, Z., Gunatilake, S., Murrary, S. B., Sheffield, J., \& Touyz, S. (2015a). Self-stigma of seeking treatment being male predict an increased likelihood of having an undiagnosed eating disorder. International Journal of Eating Disorders, 48, 775-778. doi:10.1002/eat.22413stig

Griffiths, S., Mond, J. M., Murray, S. B., \& Touyz, S. (2014). Young peoples’ stigmatising attitudes and beliefs about anorexia nervosa and muscle dysmorphia. International Journal of Eating Disorders, 47, 189-195. doi:10.1002/eat.22220

Griffiths, S., Mond, J. M., Murray, S. B., \& Touyz, S. (2015b). Positive beliefs about anorexia nervosa and muscle dysmorphia are associated with eating disorder symptomatology. Australia and New Zealand Journal of Psychiatry, 49, 812-820. doi:10.1177/0004867415572412

Hepworth, N., \& Paxton, S. J. (2007). Pathways to help-seeking in bulimia and binge eating problems: A concept mapping approach. International Journal of Eating Disorders, 40, 493-504. doi:10.1002/eat.20402

Hunt, J. S., \& Rothman, A. J. (2007). College students' mental models for recognising anorexia and bulimia nervosa. Appetite, 48, 289-300. doi:10.1016/j.appet.2006.05.013

Jones, E. E., Farina, A., Hastorf, A. H., Marcus, H., Miller, D. T., \& Scott, R. A. (1984). Social stigma: The psychology of marked relationships. New York: Freeman and Company.

Jones, W. R., Saeidi, S., \& Morgan, J. F. (2013). Knowledge and attitudes of psychiatrists toward eating disorders. European Eating Disorders Review, 21, 84-88. doi:10.1002/erv.2155

Jorm, A. F. (2000). Mental health literacy: Public knowledge and beliefs about mental disorders. British Journal of Psychiatry, 177, 396-401. doi:10.1192/bjp.177.5.396

Lauber, C., Anthony, M., Ajdacic-Gross, V., \& Rössler, W. (2004). What about psychiatrists' attitude to mentally ill people? European Psychiatry, 19, 423-427. 
doi:10.1016/j.eurpsy.2004.06.019

Mond, J., \& Arrighi, A. (2011). Gender differences in perceptions of the severity and prevalence of eating disorders. Early Intervention in Psychiatry, 5, 41-49. doi:10.1111/j.1751-7893.2010.00257.x

Mond, J., Robertson-Smith, G., \& Vetere, A. (2006). Stigma and eating disorders: Is there evidence of negative attitudes towards anorexia nervosa among women in the community? Journal of Mental Health, 15, 519-532.

doi:10.1080/09638230600902559

Penn, D. L., Guynan, K., Daily, T., Spaulding, W. D., Garbin, C. P., \& Sullivan, M. (1994). Dispelling the stigma of schizophrenia: What sort of information is best? Schizophrenia Bulletin, 20, 567-578. doi:10.1093/schbul/20.3.567

Stewart, M. C., Keel, P. K., \& Schiavo, R. S. (2006). Stigmatisation of the discrimination of anorexia nervosa. International Journal of Eating Disorders, 39, 320-325. doi:10.1002/eat.20262

Thompson-Brenner, H., Satir, D. A., Franko, D. L., \& Herzog, D. B. (2012). Clinician reactions to patients with eating disorders: A review of the literature. Psychiatric Services, 63, 73-78. doi:10.1176/appi.ps.201100050

Touyz, S. W. (2011). What kind of illness is anorexia nervosa? A clinical update. The Bulletin of the Australian Psychological Society, 33, 13-15.

Walker, S., \& Lloyd, C. (2011). Barriers and attitudes health professionals working in eating disorders experience. International Journal of Therapy and Rehabilitation, 18, 383391.

Weiss, M. G., \& Ramakrishna, J. (2006). Health-related stigma: Rethinking concepts and interventions. Psychology, Health, and Medicine, 11, 277-289. doi:10.1080/13548500600595053

Williams, M., \& Leichner, P. (2006). More training needed in eating disorders: A time cohort 
comparison study of Canadian psychiatry residents. Eating Disorders, 14, 323-334.

Wingfield, N., Kelly, N., Serdar, K., Shivy, V. A., \& Mazzeo, S. E. (2011). College students’ perceptions of individuals with anorexia and bulimia nervosa. International Journal of Eating Disorders, 44, 369-375. doi:10.1002/eat.20824 
Table 1

Participant Demographic Information.

\begin{tabular}{lccc}
\hline Variable & $\begin{array}{c}\text { Medicine } \\
(n=40)\end{array}$ & $\begin{array}{c}\text { Psychology } \\
(n=74)\end{array}$ & $\begin{array}{c}\text { Total } \\
(N=114)\end{array}$ \\
\hline Sex & $24(60.0 \%)$ & $26(35.1 \%)$ & $50(43.9 \%)$ \\
Female & $16(40.0 \%)$ & $48(64.9 \%)$ & $81(56.1 \%)$ \\
Mean age in years $(S D)$ & $25.33(6.70)$ & $22.35(7.14)$ & $23.39(5.10)$ \\
Self-reported history of AN & $1(<1.0 \%)$ & $11(9.4 \%)$ & $12(9.5 \%)$ \\
Ethnicity & & & \\
Caucasian & $20(50.0 \%)$ & $64(86.5 \%)$ & $84(73.7 \%)$ \\
Asian & $14(35.0 \%)$ & $5(6.8 \%)$ & $19(16.7 \%)$ \\
African American & $0(0.0 \%)$ & $1(<1.0 \%)$ & $1(<1.0 \%)$ \\
Pacific Islander & $0(0.0 \%)$ & $2(2.7 \%)$ & $2(1.8 \%)$ \\
Latino/Hispanic & $0(0.0 \%)$ & $1(<1.0 \%)$ & $1(<1.0 \%)$ \\
Middle Eastern & $6(15.0 \%)$ & $1(<1.0 \%)$ & $7(6.1 \%)$ \\
\hline
\end{tabular}

*Note. Participants with a self-reported lifetime history of AN were removed from the data-set, therefore the sample size reported in the table is reflective of the final sample following deletion of these data cases. 
Table 2

Means and Standard Deviations for the Dependent Variables as Function of Group and Gender.

\begin{tabular}{|c|c|c|c|c|c|}
\hline \multirow{2}{*}{$\begin{array}{l}\text { Variables } \\
\text { (including gender) }\end{array}$} & \multirow{2}{*}{$\begin{array}{c}\text { Medicine } \\
(n=40)\end{array}$} & \multirow{2}{*}{$\begin{array}{c}\begin{array}{c}\text { Psychology } \\
(n=74)\end{array} \\
M(S D)\end{array}$} & \multirow{2}{*}{$\begin{array}{c}\text { Total } \\
(N=114) \\
M(S D)\end{array}$} & \multicolumn{2}{|c|}{$\begin{array}{c}\text { Univariate Analyses } \\
\text { ( } p \text {-values })\end{array}$} \\
\hline & & & & Degree & Gender \\
\hline Blame & $3.06(.56)$ & $2.70(.70)$ & $2.83(.68)$ & ns & .007 \\
\hline Women & $2.83(.66)$ & $2.65(.71)$ & $2.68(.70)$ & & \\
\hline Men & $3.22(.43)$ & $3.11(.47)$ & $3.19(.44)$ & & \\
\hline Selfish/Vain & $2.80(.86)$ & $2.09(.81)$ & $2.34(.89)$ & .027 & ns \\
\hline Women & $2.70(.98)$ & $2.05(.85)$ & $2.17(.91)$ & & \\
\hline Men & $2.87(.78)$ & $2.43(.44)$ & $2.75(.72)$ & & \\
\hline Weak & $2.24(.73)$ & $1.89(.83)$ & $2.02(.81)$ & ns & .032 \\
\hline Women & $2.17(.66)$ & $1.80(.83)$ & $1.88(.81)$ & & \\
\hline Men & $2.29(.77)$ & $2.53(.53)$ & $2.36(.72)$ & & \\
\hline Trivilisation & $2.01(.74)$ & $1.74(.85)$ & $1.83(.82)$ & ns & .017 \\
\hline Women & $1.76(.49)$ & $1.66(.81)$ & $1.68(.76)$ & & \\
\hline Men & $2.17(.84)$ & $2.27(.99)$ & $2.19(.87)$ & & \\
\hline Total ED Stigma & $50.28(11.68)$ & $41.52(13.52)$ & $44.59(13.52)$ & ns & .009 \\
\hline Women & $47.12(12.02)$ & $40.20(13.58)$ & $41.57(13.50)$ & & \\
\hline Men & $52.38(11.22)$ & $51.00(8.90)$ & $52.00(10.52)$ & & \\
\hline Responsibility & $2.66(.75)$ & $2.38(.92)$ & $2.48(.87)$ & ns & .011 \\
\hline Women & $2.50(.86)$ & $2.28(.89)$ & $2.33(.88)$ & & \\
\hline Men & $2.77(.68)$ & $3.11(.82)$ & $2.86(.72)$ & & \\
\hline Fear \& Exclusion & $2.40(.80)$ & $2.70(.77)$ & $2.24(.77)$ & ns & ns \\
\hline Women & $2.32(.82)$ & $2.84(.83)$ & $2.19(.76)$ & & \\
\hline Men & $2.61(.73)$ & $2.60(.72)$ & $2.61(.78)$ & & \\
\hline Affective Reaction & $3.89(.83)$ & $3.43(.86)$ & $3.60(.88)$ & .038 & ns \\
\hline Women & $4.06(.95)$ & $3.41(.88)$ & $3.54(.93)$ & & \\
\hline Men & $3.77(.75)$ & $3.61(.73)$ & $3.73(.74)$ & & \\
\hline Characteristics & $4.42(.53)$ & $4.49(.59)$ & $4.47(.57)$ & ns & ns \\
\hline Women & $4.52(.59)$ & $4.47(.61)$ & $4.48(.61)$ & & \\
\hline Men & $4.35(.50)$ & $4.63(.38)$ & $4.43(.48)$ & & \\
\hline Sociocultural Attribution & $5.60(.84)$ & $5.33(.68)$ & $5.43(.68)$ & ns & .012 \\
\hline Women & $5.49(.51)$ & $5.38(.61)$ & $5.41(.59)$ & & \\
\hline Men & $5.67(.46)$ & $4.95(1.02)$ & $5.47(.72)$ & & \\
\hline Biogenetic Attribution & $4.43(1.10)$ & $4.55(1.20)$ & $4.41(1.15)$ & ns & .049 \\
\hline Women & $4.59(.84)$ & $4.65(1.18)$ & $4.64(1.11)$ & & \\
\hline Men & $3.85(1.03)$ & $3.83(1.20)$ & $3.85(1.06)$ & & \\
\hline
\end{tabular}

